\title{
Inhibition of cystic fibrosis transmembrane conductance regulator by novel interaction with the metabolic sensor AMP-activated protein kinase
}

\author{
Kenneth R. Hallows, ${ }^{1}$ Viswanathan Raghuram, ${ }^{2}$ Bruce E. Kemp, ${ }^{3}$ \\ Lee A. Witters, ${ }^{4}$ and J. Kevin Foskett ${ }^{2}$ \\ ${ }^{1}$ Renal-Electrolyte and Hypertension Division, Department of Medicine, and \\ ${ }^{2}$ Department of Physiology and Institute for Human Gene Therapy, University of Pennsylvania School of Medicine, \\ Philadelphia, Pennsylvania, USA \\ ${ }^{3}$ St. Vincent's Institute of Medical Research, Fitzroy, Victoria, Australia \\ ${ }^{4}$ Departments of Medicine and Biochemistry, Division of Endocrinology, Diabetes and Metabolism, \\ Dartmouth Medical School, Hanover, New Hampshire, USA \\ Address correspondence to: J. Kevin Foskett, Department of Physiology and Institute for Human Gene Therapy, \\ University of Pennsylvania School of Medicine, B39 Anatomy/Chemistry Building, Philadelphia, Pennsylvania 19104, USA. \\ Phone: (215) 898-1354; Fax: (215) 573-6808; E-mail: foskett@mail.med.upenn.edu. \\ Kenneth R. Hallows and Viswanathan Raghuram contributed equally to this work.
}

Received for publication February 15, 2000, and accepted in revised form May 2, 2000.

\begin{abstract}
The cystic fibrosis transmembrane conductance regulator (CFTR) is an ATP-gated $\mathrm{Cl}^{-}$channel that regulates other epithelial transport proteins by uncharacterized mechanisms. We employed a yeast two-hybrid screen using the $\mathrm{COOH}$-terminal 70 residues of CFTR to identify proteins that might be involved in such interactions. The $\alpha 1$ (catalytic) subunit of AMP-activated protein kinase (AMPK) was identified as a dominant and novel interacting protein. The interaction is mediated by residues $1420-1457$ in CFTR and by the COOH-terminal regulatory domain of $\alpha 1$-AMPK. Mutations of two protein trafficking motifs within the 38-amino acid region in CFTR each disrupted the interaction. GST-fusion protein pull-down assays in vitro and in transfected cells confirmed the CFTR- $\alpha 1$-AMPK interaction and also identified $\alpha 2$-AMPK as an interactor with CFTR. AMPK is coexpressed in CFTR-expressing cell lines and shares an apical distribution with CFTR in rat nasal epithelium. AMPK phosphorylated full-length CFTR in vitro, and AMPK coexpression with CFTR in Xenopus oocytes inhibited cAMP-activated CFTR whole-cell $\mathrm{Cl}^{-}$conductance by approximately $35-50 \%$. Because AMPK is a metabolic sensor in cells and responds to changes in cellular ATP, regulation of CFTR by AMPK may be important in inhibiting CFTR under conditions of metabolic stress, thereby linking transepithelial transport to cell metabolic state.
\end{abstract}

J. Clin. Invest. 105:1711-1721 (2000).

\section{Introduction}

Cystic fibrosis (CF), the most common lethal genetic disease among Caucasians, is complicated by abnormal epithelial solute and fluid transport due to mutations in the cystic fibrosis transmembrane conductance regulator (CFTR) (1). CFTR belongs to the ATP-binding cassette (ABC) family of transporters, containing 12 predicted transmembrane helices and five cytoplasmic domains consisting of two nucleotide-binding domains (NBDs), a regulatory (R) domain containing numerous consensus phosphorylation sequences, and $\mathrm{NH}_{2}$ - and $\mathrm{COOH}$-terminal tails $(2,3)$. CFTR is a cAMP-dependent protein kinase-activated (PKA), ATP-gated $\mathrm{Cl}^{-}$channel whose channel function is defective in CF (4). CFTR $\mathrm{Cl}^{-}$channel activity requires phosphorylation of the $\mathrm{R}$ domain and ATP binding and hydrolysis at both $\operatorname{NBDs}(5,6)$. A unique feature of CFTR is the requirement for ATP hydrolysis to support channel activity, with $\mathrm{Cl}^{-}$ions diffusing energetically downhill according to the elec- trochemical gradient. CFTR may require hydrolysis to actively transport substrates (7), although none have been identified. Alternately, the requirement for ATP hydrolysis may enable CFTR activity to be coupled to cellular metabolism $(8,9)$.

Although CF is caused by mutations in CFTR, it is unclear how or whether the consequent $\mathrm{Cl}^{-}$channel defects cause the CF phenotype per se. CFTR may also act as a "conductance regulator," coordinating an ensemble of transmembrane ion fluxes in polarized epithelia. Several plasma membrane transport proteins may be modulated by CFTR, including the epithelial $\mathrm{Na}^{+}$channel (ENaC); the outwardly rectifying $\mathrm{Cl}^{-}$channel; $\mathrm{Ca}^{2+}$-activated and volume-activated $\mathrm{Cl}^{-}$channels; the ROMK K${ }^{+}$channel; anion exchanger; and the aquaporin 3 water channel (10-15). The mechanisms involved in this modulation by CFTR are poorly understood. The conductance-regulator function of CFTR may involve ATP release from cells and autocrine activation of signaling pathways that mod- 
ulate permeation pathways $(16,17)$. CFTR might also influence activities of other transport pathways by direct or indirect protein-protein interactions. The $\mathrm{NH}_{2}$-terminus of CFTR binds syntaxin $1 \mathrm{~A}$, which reduces the magnitude of cAMP-stimulated CFTRmediated whole-cell $\mathrm{Cl}^{-}$currents (18), possibly by disrupting intramolecular interactions of the $\mathrm{NH}_{2}$-terminus with the $\mathrm{R}$ domain (19). The $\mathrm{COOH}$-terminus of CFTR ends in a sequence that comprises a PDZ domain-binding motif. The $\mathrm{Na}^{+} / \mathrm{H}^{+}$exchange regulatory factor (NHERF or EBP50) contains two PDZ domains and interacts with this motif (20). It has been speculated that NHERF functions as a scaffold in a macromolecular complex that facilitates the trafficking and localization of CFTR and, importantly, its regulation of other transport proteins (20). However, direct evidence for such a physiological role of NHERF or any other interacting proteins in vivo is currently lacking.

To identify other possible CFTR-interacting proteins, we performed a yeast two-hybrid screen using the COOH-terminal 70 amino acids of CFTR (1411-1480). In addition to identifying NHERF, we found that the $\alpha 1$ (catalytic) subunit of the adenosine 5 '-monophosphate-activated protein kinase (AMPK) was also a strong and consistent interactor. AMPK is a serine/threonine kinase that exists as an $\alpha \beta \gamma$ heterotrimer. Multiple isoforms $(\alpha 1, \alpha 2, \beta 1, \beta 2, \gamma 1, \gamma 2$, and $\gamma 3$ ) exist, with differing tissue distributions and presumed substrates (21) and homologues in all eukaryotes (22). AMPK is a metabolic sensor that responds to changes in AMP/ATP ratios, for example during conditions of nutritional stress, by phosphorylating important rate-limiting metabolic enzymes (e.g., HMG-CoA reductase and acetyl-CoA carboxylase), inhibiting their activities (22). AMPK thereby slows nonessential ATP-requiring pathways and consequently conserves cellular energy stores. More recent studies have also suggested roles for AMPK in the regulation of glucose-dependent gene expression, cellular glucose uptake, and endothelial nitric oxide synthase activity (23-25).

Here, we have mapped interacting regions and residues on both the CFTR tail and the AMPK $\alpha 1$ subunit ( $\alpha 1-\mathrm{AMPK})$ and showed that AMPK interacts with CFTR in mammalian cells. We demonstrate that AMPK is endogenously present in cells that express CFTR and colocalizes with CFTR in apical regions of epithelial tissues. Importantly, CFTR is a substrate for AMPK phosphorylation in vitro, and coexpression of AMPK with CFTR in Xenopus oocytes inhibits CFTR whole-cell $\mathrm{Cl}^{-}$conductances. Because CFTR hydrolyzes ATP and has been associated with ATP efflux from cells, the interaction of AMPK with CFTR may suggest a novel mechanism to link CFTR ion channel activity to cell metabolic status, thereby coupling cellular metabolism with transepithelial solute transport and the maintenance of cellular ion gradients.

\section{Methods}

Yeast two-hybrid analysis

Yeast two-hybrid screen. Using pLexA-CFTR, created by cloning DNA encoding the cytoplasmic tail of CFTR (amino acids 1411-1480) into pLexA, as a bait in twohybrid screening of a human testis cDNA library (CLONTECH Laboratories Inc., Palo Alto, California, USA), approximately $10^{7}$ primary transformants were pooled and selected for growth in the absence of leucine and for $\beta$-galactosidase activity on X-gal plates. Positive clones were grouped based on insert size and restriction pattern and sequenced. Sequences were compared with those in the National Center for Biotechnology Information databank using the basic local alignment search tool (26). Twelve of the 31 confirmed positive clones were $\mathrm{COOH}$-terminal fragments of $\alpha 1-A M P K$, ranging in length from amino acids 294-550 (shortest) to 161-550 (longest).

Yeast "bait" and "prey" plasmid constructs. All baits and preys were constructed in pLexA and pB42AD (CLONTECH), respectively. Inserts were constructed by PCR amplification or by subcloning required regions. Sitedirected mutagenesis was performed using two-step sequential PCR with overlapping primers. Sequences of primers used for PCR amplification are available on request. Plasmid inserts were verified by sequencing.

Two-hybrid interaction trap. EGY48[p8op-LacZ] yeast (27) was transformed with various bait and prey plasmids (28), and at least 10 transformants were streaked in replicate onto X-gal plates and leucine deficient plates to assess interaction strength in an interaction trap assay. Control yeast, transformed with either bait or prey plasmids alone, was also streaked on appropriate drop-out media plates to rule out false positives (none found). The interaction strength of each streak was graded on a scale from - to +++ on days 1,2 , and 3 after streaking on both X-gal plates (-, no blue color; +, light blue; ++, medium blue; +++, dark blue) and leucine-deficient plates (-, no growth; + , negative to minimal growth after 24 hours, ++ , significant growth after 24 hours and confluent by 72 hours; +++ , confluent growth within 24 to 48 hours). The average scores of all streaks determined on day 3 for each interaction are reported in Figure 1. For all constructs, expression of fusion proteins was confirmed by Western analysis of yeast extracts. The antibodies used were as follows: monoclonal anti-LexA (CLONTECH) for bait, monoclonal anti-hemagglutinin (HA.11) (Covance, Richmond, California, USA) for prey, and secondary antimouse IgG-HRP antibodies (Amersham Pharmacia Biotech, Piscataway, New Jersey, USA).

\section{Cell culture and transfection}

$\mathrm{CHO}$ (Chinese hamster ovary) cells and a $\mathrm{CHO}$ cell line that stably expresses CFTR (CHO-BQ2) were cultured as described (29). Cells were transfected (Lipofectamine; GIBCO BRL, Gaithersburg, Maryland, USA) using $5 \mu \mathrm{g}$ plasmid DNA and harvested 2 days later. 
Glutathione S-transferase pull-down assays

In vitro binding studies. The $\mathrm{COOH}$-terminus of CFTR (amino acids 1411-1480) was cloned into pGEX6p-1 (Amersham Pharmacia Biotech). Glutathione S-transferase-CFTR (GST-CFTR) fusion protein was expressed in Escherichia coli and affinity-purified using glutathione-Sepharose beads (GSH-Sepharose; Amersham Pharmacia Biotech). Purified rat liver AMPK holoenzyme (200 $\mathrm{ng}$ ), isolated as described (30), was rotated for $2-3$ hours at $4^{\circ} \mathrm{C}$ with $5 \mu \mathrm{g}$ of GST or GSTCFTR immobilized on GSH-Sepharose beads in lysis buffer containing PBS ( $\mathrm{pH}$ 7.4) plus 1\% Triton X-100 and $2 \mathrm{mg} / \mathrm{mL}$ BSA. Beads were washed with lysis buffer, subjected to SDS-PAGE, transferred to nitrocellulose, and immunoblotted with primary rabbit polyclonal antibodies to $\alpha 1$-AMPK (1:1000 dilution), prepared as described previously (31), followed by secondary anti-rabbit IgG-HRP antibodies (1:10,000 dilution; Boehringer-Mannheim, Indianapolis, Indiana, USA). After chemiluminescence exposure, membranes were stained with Ponceau $S$ to verify that similar amounts of GST were present under experimental and control conditions.

In vivo binding studies. CHO-BQ2 cells were transfected with various GST-tagged rat $\alpha 1$ or $\alpha 2$ catalytic subunits of AMPK cDNAs in the $\mathrm{pEBG}$ mammalian expression vector (32) 48 hours before experiments. Cells were washed in ice-cold PBS and lysed in ice-cold lysis buffer (PBS containing 1\% Triton X-100, 2 mM EDTA, 1 mM PMSF, $10 \mu \mathrm{M}$ E-64, $1 \mu \mathrm{M}$ pepstatin A, and $1 \mu \mathrm{M}$ leupeptin). Lysates were pelleted at $14,000 \mathrm{~g}$ for 20 minutes at $4{ }^{\circ} \mathrm{C}$, the supernatant removed, and total protein concentration estimated by the Bio-Rad protein assay (BioRad Laboratories Inc., Hercules, California, USA); 250-500 $\mu \mathrm{g}$ of total cellular protein at more than 2 $\mathrm{mg} / \mathrm{mL}$ was used for affinity purification. Total protein content and concentrations were kept constant among lysates. Samples were incubated with GSH-Sepharose beads on a rotator for $2-3$ hours at $4{ }^{\circ} \mathrm{C}$ to bind GST fusion proteins and any interacting proteins. The beads were washed in lysis buffer before elution in Laemmli sample buffer at $65^{\circ} \mathrm{C}$ for 10-20 minutes. SDS-PAGE, transfer, and immunoblotting were performed as described above using primary antibodies to the CFTR $\mathrm{NH}_{2}$-terminus $\left(\mathrm{A}_{2} ; 1: 5000\right)(33)$ or goat polyclonal antiGST antibodies (1:2000; Amersham Pharmacia Biotech) and secondary anti-rabbit IgG-HRP (1:10000) or antigoat IgG-HRP antibodies (1:2000; Santa Cruz Biotechnology, Santa Cruz, California, USA).

Immunohistochemistry

Organs were dissected and removed from freshly sacrificed rats, embedded in Tissue Tek OCT compound (Sakura Finetek, Torrance, California, USA) and fresh frozen on a liquid $\mathrm{N}_{2}$ /isopentane slurry. Sections (5 $\mu \mathrm{m}$ ) were cut on a cryostat (Frigocut 2800E; ReichertJung, Vienna, Austria), transferred to microscope slides, air-dried for 15-30 minutes, fixed in 4\% paraformalde- hyde for 10 minutes at room temperature, washed in PBS (twice for 5 minutes), and then used immediately for immunohistochemical staining. Anti- $\alpha 1-A M P K$ antibodies (see above) or rabbit polyclonal antibodies directed against the COOH-terminal tail of CFTR (pAbC-term.B; Genzyme Pharmaceuticals, Cambridge, Massachusetts, USA) were used. Biotinylated secondary anti-rabbit immunoglobulin and rhodamine-conjugated avidin were from Boehringer-Mannheim. For immunostaining, primary antibodies in PBS (1:100 for anti-CFTR, 1:200 for anti- $\alpha 1$-AMPK) were added for 2 hours at room temperature. After washing, secondary biotinylated anti-rabbit IgG (1:500) was added for 1 hour at room temperature. Sections were washed again in PBS, and rhodamine-conjugated avidin was added for 30 minutes at room temperature, as well as the nuclear dye 4'-6-diamidino-2-phenylindole (DAPI) at 1 $\mu \mathrm{g} / \mathrm{mL}$. After a final wash, sections were coverslipped in 1,4-diazabicyclo[2.2.2] octane (DABCO).

\section{Phosphorylation assays}

Confluent $\mathrm{CHO}-\mathrm{BQ} 2$ cells were washed in ice-cold Trisbuffered saline (150 mM NaCl, 50 mM Tris-HCl, pH 7.5) before addition of ice-cold lysis buffer (LB) containing $100 \mathrm{mM} \mathrm{NaCl}, 50 \mathrm{mM}$ Tris-HCl, $5 \mathrm{mM}$ EDTA, $50 \mathrm{mM}$ $\mathrm{NaF}, 5 \mathrm{mM}$ NaPPi, 1\% Na-deoxycholate, 1\% Triton X100 , and $0.1 \%$ SDS. Protease and phosphatase inhibitors (1 mM PMSF, $0.1 \mathrm{mg} / \mathrm{mL}$ aprotinin, $0.1 \mathrm{mM}$ EGTA, 0.1 mM Na-orthovanadate) were added to LB (LB plus inhibitors; LBI) just before lysis. Lysates were pelleted for 20 minutes at $4^{\circ} \mathrm{C}$ at $14,000 \mathrm{~g}$. One milligram of soluble lysate for each sample was reacted with a $\mathrm{COOH}$-terminal CFTR mAb (1:100, Genzyme 24-1) for 1 hour at $4{ }^{\circ} \mathrm{C}$, followed by addition of $5 \mu \mathrm{L}$ protein $\mathrm{A} / \mathrm{G}$ beads (Pierce Chemical Co., Rockford, Illinois, USA) for 1 hour to precipitate CFTR. Beads were washed twice in $4 \times$ AMPK assay buffer (containing $200 \mathrm{mM}$ Na-HEPES, $400 \mathrm{mM}$ $\mathrm{NaCl}, 4 \mathrm{mM}$ DTT, and $20 \mathrm{mM}$ Mg-acetate), then resuspended in $22 \mu \mathrm{L}$ final volume of $1 \times$ AMPK assay buffer $\left( \pm 0.2 \mathrm{mM}\right.$ AMP) containing $20 \mu \mathrm{Ci}\left[\gamma^{32} \mathrm{P}\right]$-ATP $(\mathrm{NEN}$ Life Sciences Products Inc., Boston, Massachusetts, USA) and $2 \mu \mathrm{L}$ of either affinity-purified AMPK holoenzyme, consisting of GST-tagged $\alpha 1$ and HA-tagged $\beta 1$ and $\gamma 1$ subunits (32), or constitutively-active GST-tagged $\alpha 1-1$ 312 fragment (34). Specific activities of AMPK holoenzyme and $\alpha 1-1-312$ were 0.33 and $0.55 \mathrm{pmol} / \mathrm{min} / \mu \mathrm{L}$ of isolate, respectively. Phosphorylation proceeded for 1-2 hours with gentle mixing at room temperature before washing beads twice with LBI. Beads were resuspended in $25 \mu \mathrm{L}$ Laemmli sample buffer at $37^{\circ} \mathrm{C}$ for 5 minutes to elute CFTR before loading on a $6 \%$ gel for electrophoresis. After drying the gel, autoradiography exposure for 1-2 hours was performed at room temperature.

\section{Two-electrode voltage clamp experiments using cRNA-injected Xenopus oocytes}

Maintenance of Xenopus laevis, surgical extraction of ovaries, collagenase treatment, and injection of oocytes were carried out as described (35). CFTR complemen- 
tary RNA (cRNA) was transcribed in vitro (SP6 mMessage mMachine kit; Ambion Inc., Austin, Texas, USA) using linearized PSPCFTR (36). AMPK subunit cRNAs were transcribed in vitro ( $\mathrm{T} 7 \mathrm{mMessage} \mathrm{mMachine}$ kit) using template cDNAs generated by high-fidelity PCR (PFU-Turbo Kit; Stratagene, La Jolla, California, USA) of HA-tagged subunits (32), with sense primer consisting of the T7 promoter and HA tag, and antisense primers complementary to $\mathrm{COOH}$-terminal sequences of each subunit. PCR products were verified by sequencing. The cRNA purity and quantity were assessed by agarose-gel electrophoresis. Oocytes were injected with CFTR cRNA (20 ng) plus or minus vari-

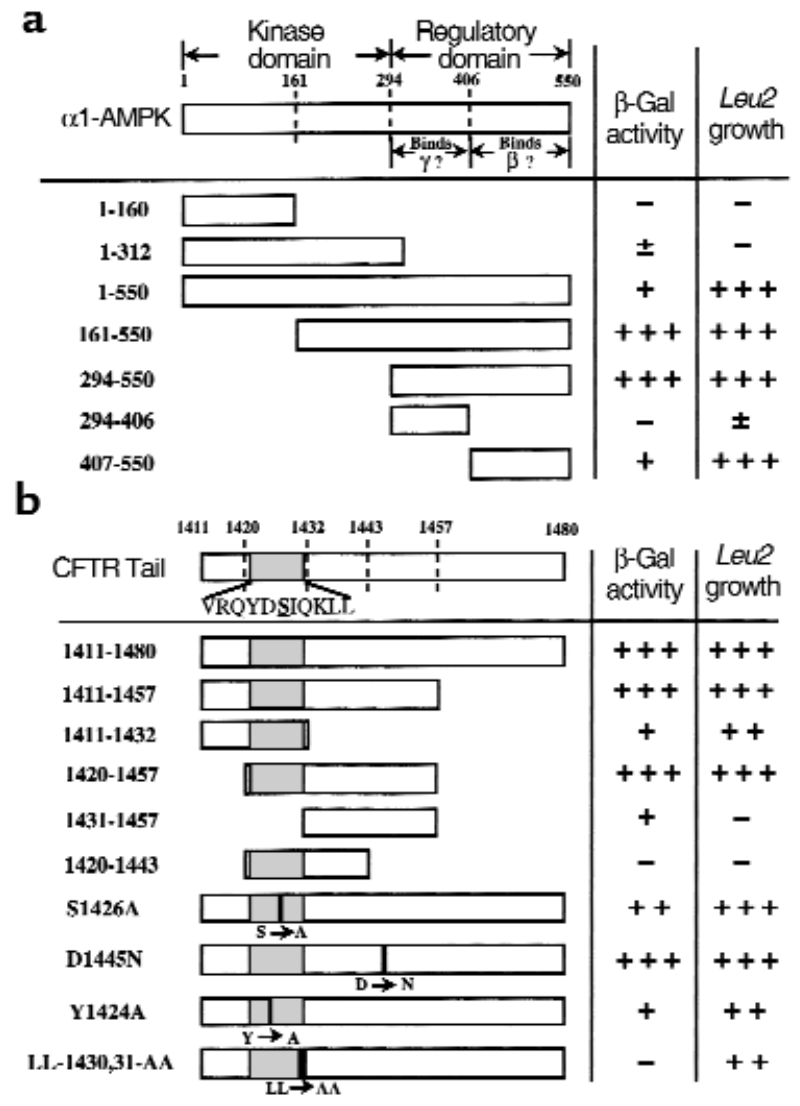

Figure 1

Yeast two-hybrid interaction maps of $\alpha 1$-AMPK and CFTR $\mathrm{COOH}$ terminal tail. (a) Determination of regions of $\alpha 1-A M P K$ important for interaction with CFTR-1411-1480. Functional regions of $\alpha 1$ AMPK are shown schematically at the top, as estimated by sequence homology to yeast Snf1p (38). Constructs containing residues upstream of amino acid 161 were made by high-fidelity PCR amplification using appropriate primers and full-length rat $\alpha 1$-AMPK cDNA (31) as template. Other constructs were either original prey clones identified in the two-hybrid screen ( $\alpha 1-161-550$ and 294-550) or were generated by PCR using $\alpha 1-294-550$ prey plasmid as a template. (b) Determination of region and specific residues within CFTR1411-1480 important for interaction with $\alpha 1-294-550$. Expression of the CFTR-1420-1443 fragment could not be detected by Western analysis. Because lack of interaction observed may have resulted from no expression, it is uncertain whether residues 1444-1457 are required for strong interaction. ous AMPK subunits of cRNA (5-15 ng total) in a total volume of $46 \mathrm{~nL}$. Oocytes, maintained as described (35), were used 2-4 days after injection. Protein expression by AMPK constructs was confirmed (TNT T7-coupled in vitro transcription/translation reticulocyte lysate kit; Promega Corp., Madison, Wisconsin, USA) with ${ }^{35} \mathrm{~S}$-methionine labeling followed by SDS-PAGE and autoradiography.

The two-electrode voltage clamp (TEV) method was used to measure single-oocyte whole-cell currents, from which whole-cell conductances were calculated (35). CFTR whole-cell $\mathrm{Cl}^{-}$conductance was stimulated by perfusing oocytes with $10 \mu \mathrm{M}$ forskolin and $1 \mathrm{mM} 3$ isobutyl-1-methylxanthine (IBMX). The change in whole-cell conductance was calculated as the difference in final steady-state conductance after CFTR stimulation compared with baseline conductance, which was generally less than $1 \%$ of the stimulated level.

Data from different oocyte batches were pooled, and statistical analyses were performed using an ANOVA factorial model (StatView; Abacus Concepts Inc., San Francisco, California, USA) to account for batch-tobatch variability in stimulated CFTR conductances.

\section{Results}

Identification of $A M P K$ as an interactor with the $\mathrm{COOH}$-terminus of CFTR by yeast two-bybrid screen. A yeast genetic screen was undertaken to identify proteins that interact with the COOH-terminus of CFTR (CFTR 1411-1480). Several different clones containing partial sequences of $\alpha 1$-AMPK (1-550) were identified in two independent assays, growth on leucine-deficient media and $\beta$-galactosidase activity. These clones, which represented approximately $40 \%$ of the total clones identified, ranged in length from residues $161-550$ to 294-550.

Mapping CFTR-1411-1480 and $\alpha 1-A M P K$ interaction determinants by yeast two-bybrid analysis. Most of the catalytic domain of $\alpha 1$-AMPK comprising the $\mathrm{NH}_{2}$-terminal half of the protein (34) was not represented in any of the clones identified in the two-hybrid screen. This suggested that the interaction of $\alpha 1$-AMPK with CFTR likely occurs through the COOH-terminal half of $\alpha 1$ AMPK, which is involved in binding the $\beta$ and $\gamma$ regulatory subunits (Figure 1a, top) (37). Truncation mutants of $\alpha 1$-AMPK were constructed to assess the regions responsible for the CFTR interaction (Figure 1a). Fragments corresponding to the $\mathrm{NH}_{2}$-terminal kinase domain interacted weakly, if at all (compare fragments $1-160$ and 1-312). Within the regulatory domain, the COOH-terminal half (residues 407-550) bound more tightly to the CFTR tail. Based on alignment with the yeast SNF1 sequence (38), this region may be involved in $\beta$ subunit binding (Figure 1a, top).

To identify interaction determinants within the CFTR tail, truncation and point mutants were tested for their ability to interact with the minimally sufficient $\alpha 1-294-550$ interacting fragment (Figure 1b). Similar results were obtained with the longer $\alpha 1-161$ 550 interacting fragment (not shown). The minimum 
CFTR fragment sufficient for strong interaction with $\alpha-1$-AMPK included residues $1420-1457$. Within this region there exist possible consensus sequences for AMPK phosphorylation, including one centered on Ser at 1426 (residues 1421-1431; filled portion of the bar in Figure 1b) (22). However, mutation of S1426 (S1426A and S1426D [not shown]) had no effect on the interaction strength. Mutation of Asp at 1445 to Asn (D1445N), a CF-causing mutation (Cystic Fibrosis Mutation Database at http://www.genet.sickkids.on.ca/cftr) also had no effect. A recent study suggested that Tyr at 1424 (Y1424) is important for endocytic internalization of CFTR (39). Interestingly, the mutation of Y1424A substantially diminished the interaction strength. Mutation of another possible trafficking motif in the vicinity, the di-leucine at
1430 and 1431 (40) (LL-1430,1431-AA) also dramatically reduced the interaction strength.

Confirmation of the AMPK-CFTR interaction using GST pull-down assays. The AMPK-CFTR interaction was examined using GST fusion protein-affinity purification (pull-down) assays performed in vitro and in vivo. For in vitro pull-down assays, either purified GSTCFTR-1411-1480 fusion protein or GST alone was immobilized on GSH-Sepharose beads in vitro, and purified rat liver AMPK holoenzyme was added. The $\alpha 1$ was pulled down in the presence of the GST-CFTR1411-1480 but not with GST alone (Figure 2a), confirming that $\alpha 1$-AMPK interacts directly and specifically with the CFTR COOH-terminal tail in vitro.

To determine if the $\alpha 1$ subunit interacts with CFTR in mammalian cells, CHO-BQ2 cells were transiently a

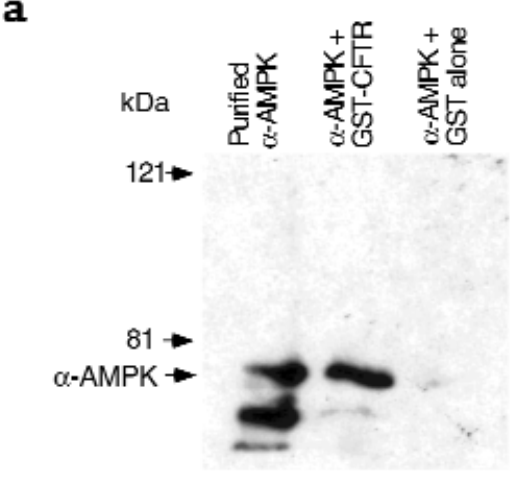

C

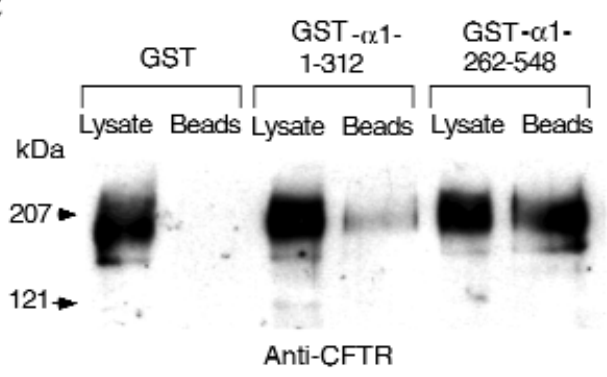

Anti-CFTR

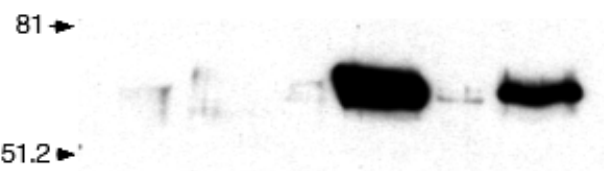

$33.6-$ b

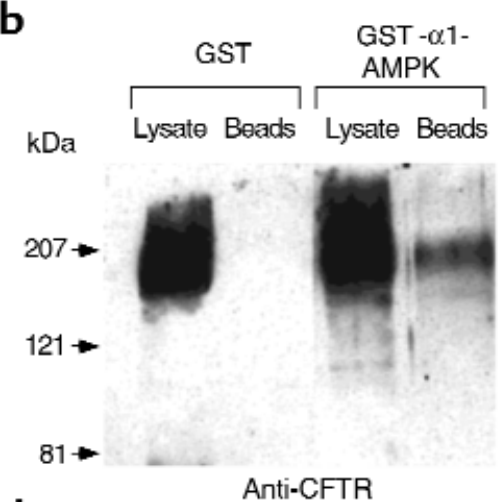

d

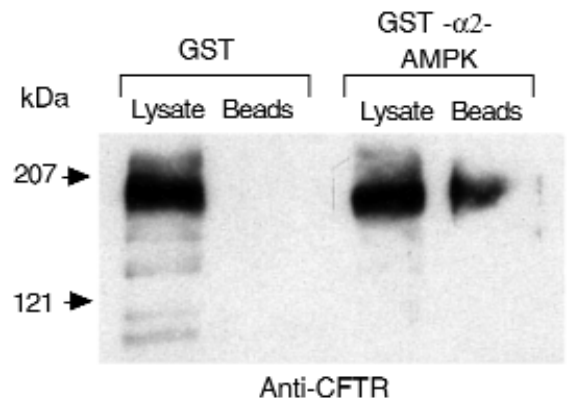

\section{Anti-GST}

\section{Figure 2}

Confirmation of AMPK-CFTR interaction by GST pull-down assays. (a) CFTR COOH-terminal tail (GST-CFTR-1411-1480) pulls down $\alpha 1$-AMPK in vitro. Purified liver AMPK holoenzyme (15 ng) was loaded in first lane as reference. (b) GST- $\alpha 1$-AMPK fusion protein expressed in CHO-BQ2 cells pulls down full-length CFTR in vivo. Ten micrograms of the total soluble cellular protein was loaded in first and third (lysate) lanes as reference for CFTR. Second and fourth (beads) lanes show eluate from GSH-Sepharose beads after affinity purification and washing. (c) Comparison of binding strengths of CFTR with $\mathrm{NH}_{2}$-terminal and $\mathrm{COOH}$-terminal GST- $\alpha 1$-AMPK fusion proteins in $\mathrm{CHO}-\mathrm{BQ} 2$ cells. Lower panel is the same membrane probed with anti-GST antibodies to allow comparisons of different CFTR bands in panel above. (d) GST- $\alpha 2$-AMPK fusion protein expressed in CHO-BQ2 cells pulls down CFTR in vivo. All results shown are representative of at least three replicate experiments. 


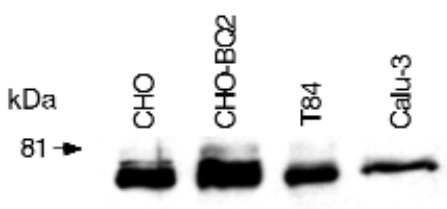

$51-$

\section{Figure 3}

Western blot of lysates from various cell lines. Total protein (10-20 $\mu \mathrm{g}$ per lane) was loaded on a $10 \%$ gel and immunoblotted with anti- $\alpha 1$-AMPK antibody $(1: 1,000)$, followed by anti-rabbit IgG$\operatorname{HRP}(1: 10,000)$.

transfected with GST- $\alpha 1$-AMPK constructs or GST alone. GST fusion proteins in cellular lysates were affinity purified and immunoblotted for CFTR (Figure 2 , b and c). CFTR was pulled down in the presence of $\alpha 1$, but not with GST alone, confirming that $\alpha 1$ specifically interacts with CFTR in these cells (Figure 2b). Consistent with the two-hybrid results, GST- $\alpha 1-262-$ 548 interacted more strongly than did GST- $\alpha 1-1-312$ (Figure 2c, top), particularly when compensation is made for the greater expression of GST- $\alpha 1-1-312$ (Figure $2 \mathrm{c}$, bottom). These results confirm that $\alpha 1$ interacts with CFTR in mammalian cells, predominantly through the $\mathrm{COOH}$-terminal half of the $\alpha 1$ subunit. Similar experiments using the full-length $\alpha 2$ isoform (Figure 2d) indicate that both known $\alpha$ isoforms interact with CFTR. Because the initial two-hybrid screens detected the $\alpha 1$ isoform, and it may be the more widely distributed isoform (31), we focused in subsequent experiments on the $\alpha 1-$ CFTR interaction.

Expression and colocalization of $\alpha 1-A M P K$ and CFTR in cells and tissues. As a prerequisite for establishing physiological relevance of the observed interaction between CFTR and AMPK, both proteins should be coexpressed in the same cells and share overlapping subcellular distributions. AMPK is expressed in a wide variety of dif- ferent tissues and cells (31). To confirm its expression in cells that express CFTR, $\alpha 1$-AMPK immunoblots were performed on lysates from different cell lines. Expression was observed in CFTR-expressing colonic (T84) and lung (Calu-3) epithelial cell lines, as well as in CFTR-expressing and wild-type CHO cells (Figure 3). Immunohistochemistry of rat nasal mucosa revealed a distinctly apical distribution of CFTR only in particular epithelial surface regions in sections containing surface epithelium, ducts, and submucosal glands (Figure $4 a)$. We found $\alpha 1-A M P K$ was localized diffusely throughout the epithelial cells, consistent with a cytoplasmic distribution. In addition, a pronounced staining was associated with epithelial cell plasma membranes, including the apical membrane (Figure 4b). The more ubiquitous distribution of $\alpha 1$-AMPK and its plasma membrane localization resulted in its colocalization with CFTR wherever CFTR was present. Similar plasma membrane staining, including pronounced apical membrane localization, of AMPK exists in other CFTR-expressing epithelial tissues, including rat pancreas, ileum, trachea, and submandibular glands, as well as human sweat glands (K.R. Hallows et al., manuscript in preparation). The findings that $\alpha 1$-AMPK and CFTR are coexpressed in the same cells and share apical colocalization in surface epithelium are consistent with the idea that the proteins could interact in vivo. AMPK phosphorylates CFTR in vitro. To determine the physiological consequences of the CFTR interaction with AMPK, we first considered whether CFTR could serve as a substrate for AMPK phosphorylation. CFTR immunoprecipitated from $\mathrm{CHO}-\mathrm{BQ} 2$ cell lysates was specifically phosphorylated by affinity-purified AMPK holoenzyme (Figure 5, lane 3), which was markedly enhanced by $0.2 \mathrm{mM}$ AMP, a physiological activator of AMPK in vivo (lane 4). A constitutively active fragment of the catalytic subunit (34), $\alpha 1$-AMPK-1-312, also phosphorylated CFTR (lane 5). Additional bands present at approximately $90-95 \mathrm{kDa}$ (in lanes 3 and 4) like-
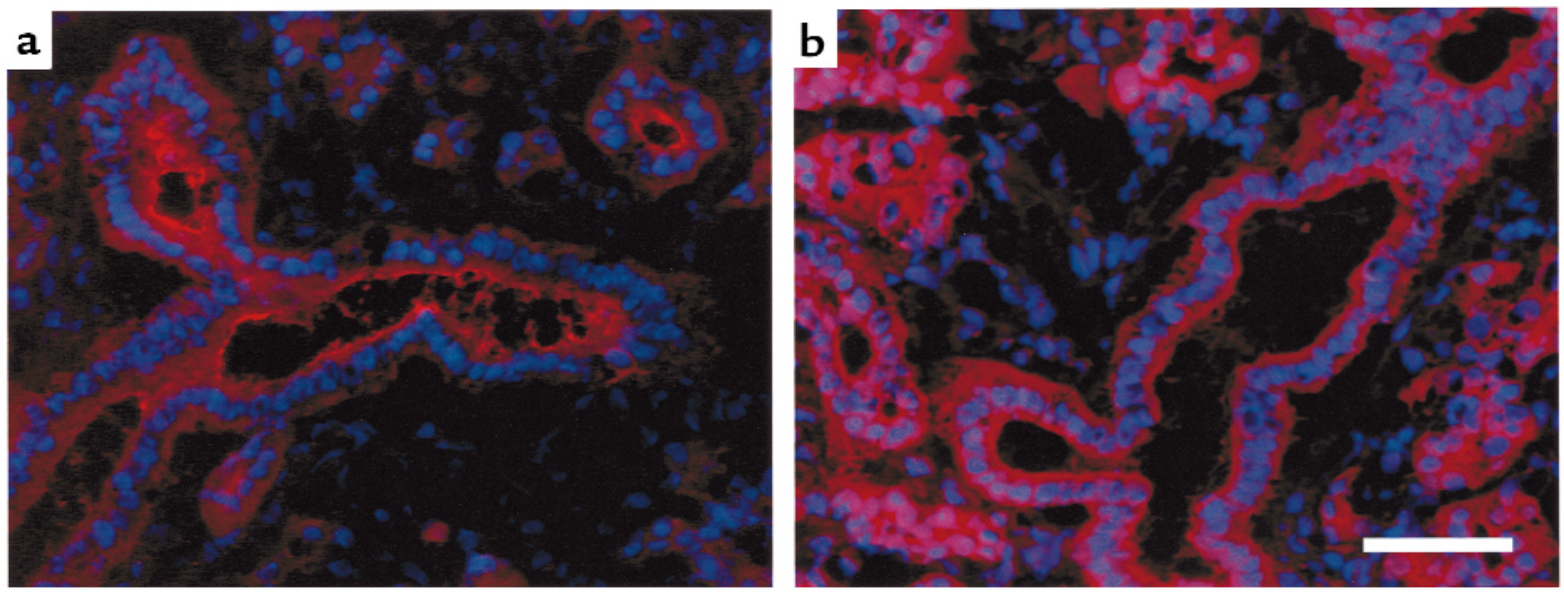

\section{Figure 4}

Immunohistochemistry of tissue sections from rat nasal mucosa. Contiguous sections were stained using (a) anti-CFTR or (b) anti- $\alpha 1$-AMPK antibodies, followed by biotinylated secondary antibodies and rhodamine-avidin. Both sections are also DAPI-nuclear stained (blue). Bar, $100 \mu \mathrm{m}$. Additional contiguous sections stained without addition of primary antibody revealed little nonspecific staining (not shown). 


\section{Figure 5}

In vitro phosphorylation of CFTR by AMPK. CFTR was immunoprecipitated from $\mathrm{CHO}-\mathrm{BQ} 2$ cell lysates (lanes 2-5) before phosphorylation, SDS-PAGE, and autoradiography. In lane 2, no exogenous kinase was added to the phosphorylation buffer. In lanes 3 and 4 , affinity-purified AMPK holoenzyme was added to immunoprecipitated CFTR in the absence or presence of $0.2 \mathrm{mM}$ AMP, respectively. Affinity-purified $\alpha 1-1-312$ was added to immunoprecipitate in lane 5. As a negative control, AMPK holoenzyme was added to an immunoprecipitate from $\mathrm{CHO}$ cells that do not express CFTR (lane $1)$. Results shown are representative of three replicate experiments.

ly represent autophosphorylated GST- $\alpha 1$-AMPK that was bound to CFTR, since the enzyme is known to autophosphorylate (41). CFTR was phosphorylated to a small extent in the absence of exogenous AMPK (lane 2 ), suggesting that endogenous kinases (e.g., AMPK and/or PKA) are coimmunoprecipitated with it or that it has some inherent autophosphorylation activity. No phosphorylation band was observed when similar protocols were performed using CHO cells lacking CFTR (lane 1). These results demonstrate that CFTR is a substrate for AMPK-dependent phosphorylation in vitro.

AMPK inbibits CFTR conductance. To determine whether AMPK affects CFTR $\mathrm{Cl}^{-}$channel activity in vivo, TEV experiments were performed in Xenopus oocytes that had been injected with CFTR cRNA alone or together with AMPK cRNAs. Changes in cAMPstimulated CFTR $\mathrm{Cl}^{-}$conductances were recorded (Figure 6). Representative conductance traces from experiments in which CFTR was expressed in the presence and absence of AMPK holoenzyme ( $\alpha 1, \beta 1, \gamma 1$ subunits together) are shown (Figure 6a). AMPK holoenzyme reduced the stimulated CFTR $\mathrm{Cl}^{-}$conductance by approximately $35 \%(P<0.001, n=27)$ (Figure $6 \mathrm{~b})$. Coexpression of just the $\alpha 1$ subunit also markedly inhibited CFTR conductance by approximately 50\% ( $P=$ $0.011, n=13)$. In contrast, coexpression of $\alpha 1-1-312$ alone, which has kinase activity but does not bind CFTR well (Figures 1a and 2c), had no effect, suggesting that AMPK kinase activity alone is not sufficient to inhibit CFTR. To test whether kinase activity is necessary for CFTR inhibition, CFTR was coexpressed with $\alpha 1-\mathrm{K} 45 \mathrm{R}$, alone or together with $\beta 1$ and $\gamma 1$. This kinase-inactive (32) mutant full-length $\alpha 1$ subunit is predicted to bind CFTR since the mutated region is not involved in the CFTR interaction. No inhibition was observed (Figure 6b), demonstrating that kinase activity is necessary and that binding of $\alpha 1$ to CFTR alone is not sufficient for CFTR inhibition. As a further control for possible effects of extra cRNA injection, coexpression of $\gamma 1$ had no effect on CFTR conductance. Thus, coexpression of AMPK holoenzyme or $\alpha 1$-AMPK alone with CFTR substantially inhibited the $\mathrm{CFTR} \mathrm{Cl}^{-}$ conductance in oocytes. Both an active kinase domain as well as the $\mathrm{COOH}$-terminal CFTR-binding sequence in $\alpha 1$-AMPK are required to confer this inhibition.

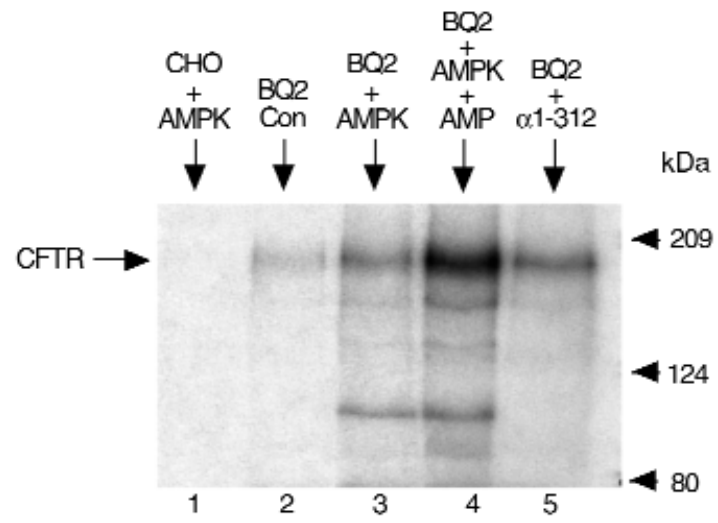

\section{Discussion}

The discovery of CFTR as the gene product mutated in $\mathrm{CF}$ and its identification as a PKA-regulated $\mathrm{Cl}^{-}$channel have provided important insights into its role in transepithelial fluid and salt transport and into the bases for channel dysfunction in the disease. Nevertheless, many pathophysiological and cell-biological features associated with expression of normal and mutant CFTR remain unexplained. The activities of several membrane transport proteins are affected by expression of CFTR, which may have significant physiological consequences, but the molecular mechanisms involved are unknown. Furthermore, the detailed mechanisms involved in the subcellular trafficking of CFTR are still unclear, despite their fundamental importance, since most cases of CF are caused by mislocalization of CFTR (42). We have considered that identification of proteinprotein interactions involving CFTR might provide new insights into important cellular processes involving CFTR. Accordingly, we initiated yeast two-hybrid screens to identify potential interacting proteins with CFTR. Here, we identified the catalytic $(\alpha)$ subunit of AMPK as the dominant interactor with the carboxy-terminal 70 amino acids of CFTR.

AMPK is a ubiquitous serine/threonine protein kinase, most likely existing in cells as a heterotrimer comprised of a catalytic $\alpha$ subunit and regulatory $\beta$ and $\gamma$ subunits (37). The $\alpha$ subunit contains an $\mathrm{NH}_{2}$-terminal catalytic domain and a $\mathrm{COOH}$-terminal regulatory domain involved in interactions with $\beta$ and $\gamma$ subunits. The kinase activity increases during conditions of metabolic stress, in response to elevated intracellular AMP/ATP ratio (37). Activation of the kinase involves both the binding of AMP to an allosteric site, probably in the $\alpha$ subunit (43), and phosphorylation by an upstream AMPKK (37). Kinase activity is also regulated by association of the $\beta$ and $\gamma$ subunits, binding of which relieves an auto-inhibitory interaction between the catalytic and regulatory domains of the $\alpha$ subunit (34). The known substrates of AMPK are relatively few, but include key enzymes in metabolic and signaling pathways. Phosphorylation by AMPK regulates their activities, acting to preserve or restore cellular ATP stores. Consequently, AMPK is believed to be a metabolic sensor in cells, responding to cellular energy state by regu- 


\section{Figure 6}

Cyclic AMP-activated CFTR whole-cell conductances in Xenopus oocytes in the presence or absence of AMPK subunits. Oocytes were injected with $20 \mathrm{ng} C F T R$ cRNA $\pm 5-15 \mathrm{ng}$ total of AMPK subunits. Two-electrode voltage clamp measurements were performed 2-4 days after injections. (a) Representative traces of CFTR $\mathrm{Cl}^{-}$conductance activation in presence or absence of coexpressed AMPK holoenzyme. Dashed vertical line indicates when oocytes were perfused with solution containing $10 \mu \mathrm{M}$ forskolin plus $1 \mathrm{mM}$ IBMX to stimulate CFTR $\mathrm{Cl}^{-}$conductance. $\mu \mathrm{S}$, microsiemens. (b) Relative stimulated CFTR conductances in oocytes expressing CFTR plus different AMPK subunits. Bars represent the mean change in conductance $( \pm$ SEM) of voltage-clamped oocytes expressing CFTR with indicated AMPK subunits relative to that of oocytes expressing CFTR alone from the same batch (horizontal dashed line at $100 \%)$. Number of replicates for each condition and $P$ values compared with the CFTR alone condition are shown. a
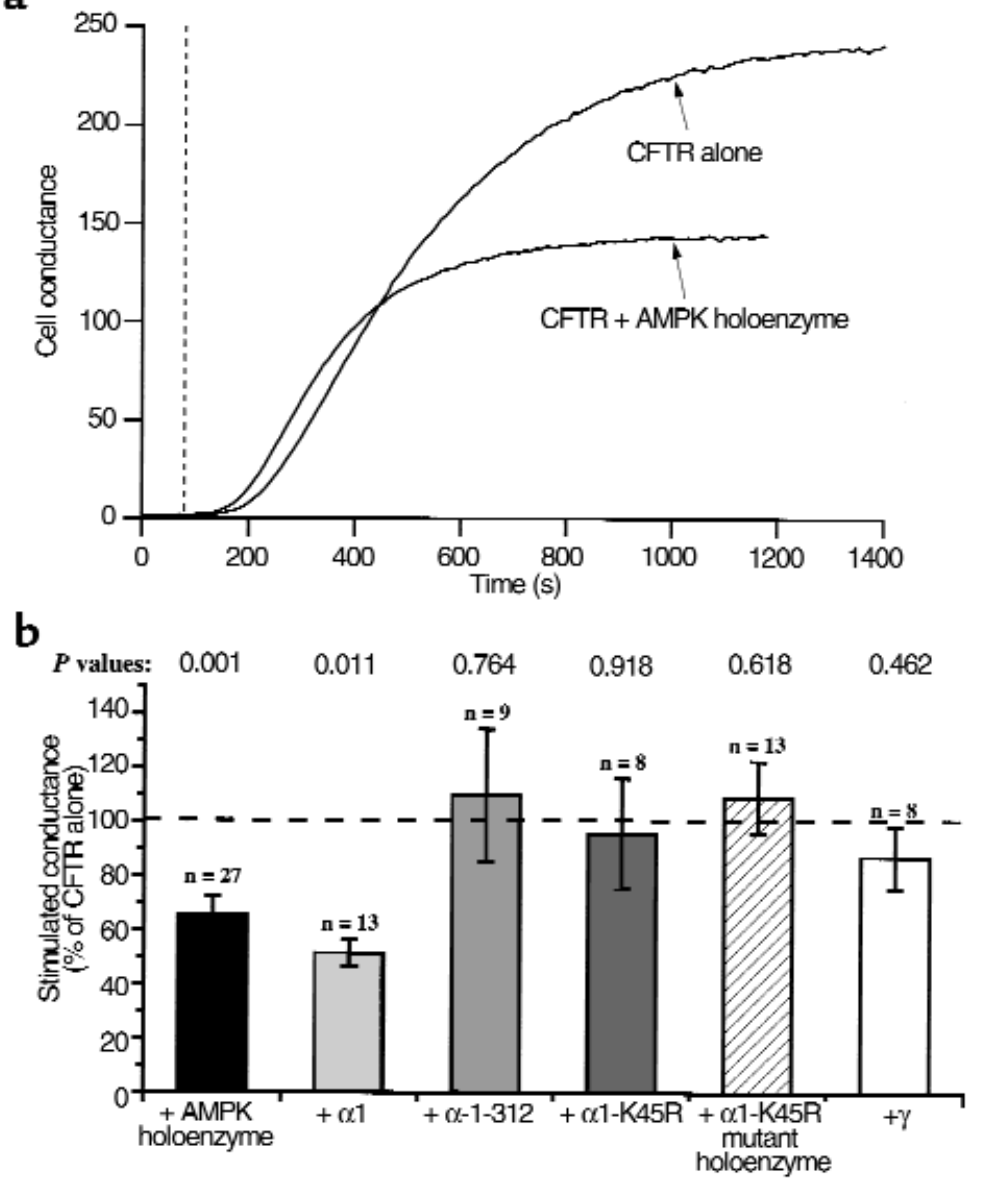

lating ATP-utilizing pathways (37). It is of interest, therefore, that AMPK associates with CFTR, an ion channel that consumes ATP during its gating and that is regulated by its phosphorylation state.

Our results demonstrate that the $\alpha 1$ subunit of AMPK interacts with CFTR in vitro as well as in vivo in both yeast and mammalian cells. GST pull-down studies indicate that the $\alpha 2$ isoform also interacts with CFTR. The interaction between $\alpha 1$ and CFTR is probably direct, since the yeast two-hybrid studies demonstrated that fragments of $\alpha 1$ alone were sufficient to interact with CFTR, and purified AMPK holoenzyme interacted with CFTR in vitro. The CFTR tail binds to $\alpha 1$ in the $\mathrm{COOH}$-terminal regulatory domain, in a region that overlaps the $\gamma$ - and $\beta$-binding regions, particularly the putative $\beta$-binding region. Nevertheless, the relationship in vivo between the binding of $\alpha$ either to CFTR or to $\beta$ and $\gamma$ remains to be determined. It is possible that the interaction of $\alpha$ with CFTR precludes $\beta$ and/or $\gamma$ binding or that the trimeric complex can associate with CFTR. The $\beta$ subunit likely provides docking sites for $\alpha$ and $\gamma$ in the kinase complex and targets it to discrete intracellular locations (37). It is interesting to speculate, therefore, that CFTR may participate as a $\beta$ surrogate, docking and localizing the $\alpha$ subunit in the immediate proximity of CFTR and nearby proteins.

The region within CFTR that interacts with $\alpha$-AMPK was refined in two-hybrid studies to a sequence encompassing amino acids $1420-1457$ within the 1480 amino acids of CFTR. The interacting region in CFTR is nearby but upstream of the carboxy-terminal residues that interact with PDZ domain-containing proteins. This region contains a known CF-causing mutation (D1445N), as well as a consensus sequence for AMPK phosphorylation centered at S1426, but neither residue appears to be important for binding. Interestingly, the 38 amino acid-interacting region in CFTR contains two distinct possible protein-trafficking motifs, Y1424 and LL1430,1431, and both are required for the interaction with $\alpha 1-$ AMPK. The Y1424 residue has been implicated in CFTR endocytosis and interactions with endocytic machinery $(39,44)$. The di-leucine motif has not been directly examined in this regard. Of note, however, the homologous di-leucine sequence plays a critical role in the mechanisms that regulate the trafficking of another $\mathrm{ABC}$ transporter, the sulfonylurea receptor, from the endoplasmic reticulum to the plasma membrane (45). The strong dependence of the interaction of CFTR with $\alpha$-AMPK on both sequences may suggest an important role of the interaction in regulating CFTR trafficking through the secretory pathway and/or at the plasma membrane.

Several observations suggest that the observed interaction of CFTR with $\alpha 1$-AMPK may be physiologically relevant. First, the interaction was detected in two dis- 
tinct assay systems. Second, both proteins are endogenously coexpressed in the same cell types in culture and in rat tissues, and $\alpha 1$-AMPK localizes, in part, to the apical membrane region in cells that express CFTR. Third, CFTR is a substrate for phosphorylation by $\alpha 1$-AMPK. CFTR contains several consensus AMPK phosphorylation sequences, but determination of the stoichiometry of phosphorylation and identification of the specific site(s) in CFTR that are phosphorylated by AMPK both in vitro and in vivo will require future studies. Fourth, coexpression of AMPK with CFTR in Xenopus oocytes caused a marked inhibition, by up to $50 \%$, in the magnitude of the cAMP-activated CFTR $\mathrm{Cl}^{-}$conductance. Finally, in preliminary experiments we have observed that treatment of polarized Calu- 3 cell monolayers with 5-aminoimidazole-4-carboxamide ribonucleoside (AICAR), a cell-permeant nucleoside analogue that pharmacologically activates AMPK in vivo (46), inhibits endogenous CFTR-dependent short-circuit currents (K.R. Hallows and J.K. Foskett, unpublished data).

Both $\alpha 1$-AMPK binding to CFTR and kinase activity appear to be required for CFTR inhibition. Thus, coexpression of either the $\alpha 1-1-312$ truncated subunit, which has constitutive kinase activity but does not bind well to CFTR, or the full-length $\alpha 1-\mathrm{K} 45 \mathrm{R}$ mutant, which lacks kinase activity but binds to CFTR, failed to inhibit CFTR $\mathrm{Cl}^{-}$conductance. Although CFTR was phosphorylated by AMPK in vitro, it remains to be determined whether the kinase requirement of $\mathrm{Cl}^{-}$conductance inhibition reflects phosphorylation of CFTR or of other substrates. AMPK inhibited CFTR activity in oocytes with normal metabolic status and in the absence of exogenously expressed $\beta$ and $\gamma$ subunits. Association of expressed $\alpha 1$-AMPK with endogenous Xenopus $\beta$ and $\gamma$ subunits, or with CFTR directly (mimicking the relief of autoinhibition, as is the case for $\beta$ and $\gamma$ binding; ref. 34), might enhance its kinase activity, although we lack data related to these possibilities.
Alternatively, kinase activity of the $\alpha$ subunit alone may be sufficient for CFTR inhibition, due to either overexpression in oocytes or to localization by docking to CFTR. The latter mechanism would be analogous to that suggested for the $G$ protein-coupled receptor kinase 6A-dependent phosphorylation of NHERF (47). Docking of AMPK to CFTR might also facilitate AMPK phosphorylation of other proteins that interact with or are near to CFTR, in a manner analogous to the adaptor function of A-kinase anchoring proteins, which contain distinct domains to bind PKA regulatory subunit and dock the complex at specific intracellular compartments (48). Regardless of the mechanisms involved, our results indicate that the oocyte environment provides for sufficient kinase activity to enable expressed AMPK to inhibit CFTR $\mathrm{Cl}^{-}$conductance.

The mechanisms by which AMPK inhibits CFTR are currently unclear, although two general possibilities include alteration of its channel properties (open probability and conductance) or alteration of the amount of CFTR at the cell surface. Activation by PKA-mediated phosphorylation is caused by changes in CFTR open probability (49). Interestingly, phosphorylation of a subset of PKA sites in CFTR inhibits channel gating (50). An analogous mechanism may be employed by AMPK, either directly by phosphorylating CFTR or indirectly through phosphorylation of a regulator(s) of CFTR gating. PKA may also activate $\mathrm{CFTR}^{-}{ }^{-}$conductance by increasing the number of active channels in the plasma membrane $(51,52)$. The possibility that AMPK regulates CFTR by decreasing its plasma membrane expression is particularly enticing since the AMPK-interacting sequence in CFTR contains two motifs involved in protein trafficking, $\mathrm{Y} 1424$ and LL1430,1431 , both of which appear to be critical for the interaction. Furthermore, the 38-amino acid AMPKinteracting region in CFTR is contained within a 44-amino acid region that is essential for stability of

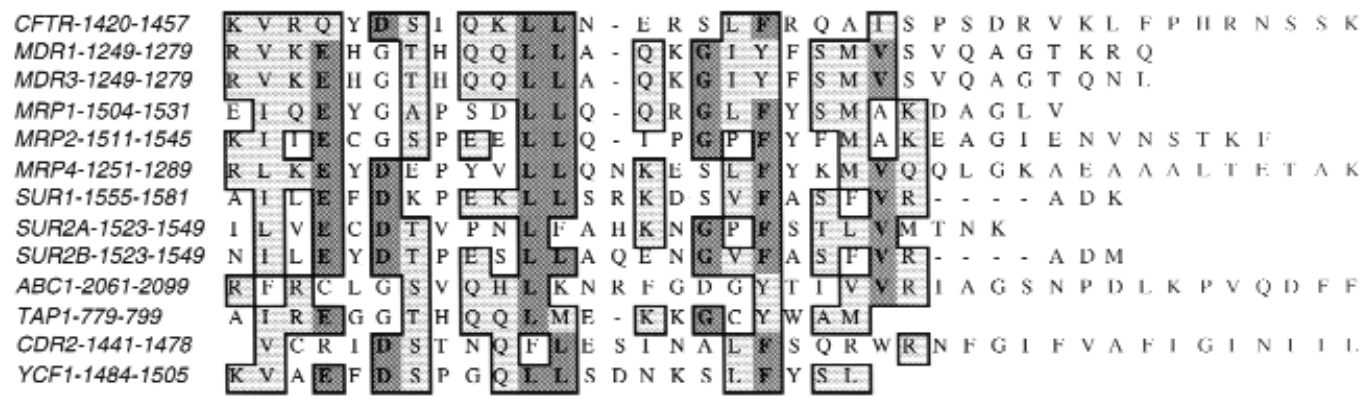

\section{Figure 7}

ClustalW alignment of peptide sequences from various $A B C$ transporters to the $\alpha$-AMPK-interacting region of the CFTR COOH-terminal tail. All sequences shown are human, except CDR2 and YCF1, which are yeast. A consensus sequence for AMPK phosphorylation [Hyd-(Basic, X)$\mathrm{X}-\mathrm{X}-\mathrm{S} / \mathrm{T}-\mathrm{X}-\mathrm{X}-\mathrm{X}$-Hyd] $(30,37)$ is preserved in $\mathrm{COOH}$-termini of MDR, ABC1, TAP1, and CDR2. GenBank accession numbers for sequences shown are: CFTR (P13569); MDR1 (P08183), MDR3 (P21439); MRP1 (P33527), MRP2 (Q92887), MRP4 (NP 005836); SUR1 (Q09428), SUR2A (AAC16057), SUR2B (AAC16058); ABC1 (NP 005493); TAP1 (A41538); CDR2 (P78595); and YCF1 (NP 010419). MDR, multidrug resistance protein; MRP, multidrug resistance-associated protein; MDP, multidrug resistance protein; SUR, sulfonylurea receptor; ABC1, ATP-binding cassette transporter 1; TAP1, transporter for antigen processing 1; CDR2, multidrug resistance protein; YCF1, metal resistance protein. 
mature complex-glycosylated CFTR (53). Thus, it is conceivable that AMPK binding to CFTR and/or phosphorylation of CFTR or nearby substrates influences the trafficking to or from the cell surface or the half-life of CFTR at the plasma membrane. Of note, ATP depletion in human airway epithelium, mimicking ischemia, a condition expected to be associated with enhanced AMPK activity, induces loss of apical membrane CFTR expression and $\mathrm{Cl}^{-}$secretion (54).

Regardless of the specific mechanisms involved, it is of interest to note that inhibition of CFTR by AMPK is predicted to reduce CFTR-dependent ATP utilization (by hydrolysis and possibly efflux), a consequence consistent with its known effects on other substrates. The region within CFTR that interacts with AMPK is well conserved among other ATP-utilizing ABC transporters (Figure 7). Of particular note, the di-leucine motif important for the AMPK-CFTR interaction is well conserved, and a consensus sequence for AMPK phosphorylation is preserved in the $\mathrm{COOH}$-termini of several members of this transporter family. These observations raise the possibility that AMPK might bind to and/or phosphorylate other $\mathrm{ABC}$ transporters in addition to CFTR. Future studies of the interactions of AMPK with transport proteins may provide insights into the mechanisms that couple metabolism to the maintenance of cellular ion gradients and to the coordination of solute transport across apical and basolateral membranes in polarized epithelial cells.

\section{Acknowledgments}

We thank J. Tazelaar for assistance with immunohistochemistry, B. Skach for the A2 CFTR antibody and helpful discussions, J. Marshall for the Genzyme pAbCterm.B antibody, J. Riordan for CFTR-transfected CHO cells, B. Reenstra for advice on phosphorylation assays, and D. Mak for assistance with TEV experiments. The work was supported by grants from the National Heart Foundation and the National Health and Medical Research Council of Australia to B.E. Kemp; National Institutes of Health (NIH), DK-35712 to L.A. Witters; and the Cystic Fibrosis Foundation, CFFS886 and FOSKET96PO. K.R. Hallows was supported by NIH National Research Service Award grants 5T32-DK07006 and F32-DK-09994-01. B.E. Kemp is an NHMRC Fellow.

1. Tsui, L.-C., and Buchwald, M. 1991. Biochemical and molecular genetics of cystic fibrosis. In Advances in buman genetics. Volume 20. H. Harris and K. Hirschhorn, editors. Plenum Press. New York, New York, USA. 153-266.

2. Rommens, J.M., et al. 1989. Identification of the cystic fibrosis gene: chromosome walking and jumping. Science. 245:1059-1065.

3. Riordan, J.R., et al. 1989. Identification of the cystic fibrosis gene: cloning and characterization of complementary DNA. Science. 245:1066-1072.

4. Welsh, M.J., and Smith, A.E. 1993. Molecular mechanisms of CFTR chloride channel dysfunction in cystic fibrosis. Cell. 73:1251-1254.

5. Cheng, S.H., et al. 1991. Phosphorylation of the R domain by cAMPdependent protein kinase regulates the CFTR chloride channel. Cell. 66:1027-1036

6. Carson, M.R., Travis, S.M., and Welsh, M.J. 1995. The two nucleotidebinding domains of cystic fibrosis transmembrane conductance reg- ulator (CFTR) have distinct functions in controlling channel activity. J. Biol. Chem. 270:1711-1717

7. Wine, J.J. 1995. Cystic fibrosis: how do CFTR mutations cause cystic fibrosis? Curr. Biol. 5:1357-1359.

8. Quinton, P.M., and Reddy, M.M. 1992. Control of CFTR chloride conductance by ATP levels through non-hydrolytic binding. Nature. 360:79-81.

9. Foskett, J.K. 1998. ClC and CFTR chloride channel gating. Annu. Rev. Physiol. 60:689-717.

10. Stutts, M.J., Rossier, B.C., and Boucher, R.C. 1997. Cystic fibrosis transmembrane conductance regulator inverts protein kinase Amediated regulation of epithelial sodium channel single channel kinetics. J. Biol. Chem. 272:14037-14040.

11. Gabriel, S.E., Clarke, L.L., Boucher, R.C., and Stutts, M.J. 1993. CFTR and outwardly rectifying chloride channels are distinct proteins with a regulatory relationship. Nature. 363:263-266.

12. Vennekens, R., et al. 1999. Inhibition of volume-regulated anion channels by expression of the cystic fibrosis transmembrane conductance regulator. J. Physiol. 515:75-85.

13. McNicholas, C.M., et al. 1996. Sensitivity of a renal $\mathrm{K}^{+}$channel (ROMK2) to the inhibitory sulfonylurea compound glibenclamide is enhanced by coexpression with the ATP-binding cassette transporter cystic fibrosis transmembrane regulator. Proc. Natl. Acad. Sci. USA. 93:8083-8088.

14. Lee, M.G., et al. 1999. Regulation of $\mathrm{Cl}^{-} / \mathrm{HCO}_{3}{ }^{-}$exchange by cystic fibrosis transmembrane conductance regulator expressed in $\mathrm{NIH}$ 3 T3 and HEK 293 cells. J. Biol. Chem. 274:3414-3421.

15. Schreiber, R., Nitschke, R., Greger, R., and Kunzelmann, K. 1999. The cystic fibrosis transmembrane conductance regulator activates aquaporin 3 in airway epithelial cells. J. Biol. Chem. 274:11811-11816.

16. Schwiebert, E.M., et al. 1995. CFTR regulates outwardly rectifying chloride channels through an autocrine mechanism involving ATP. Cell. 81:1063-1073.

17. Devidas, S., and Guggino, W.B. 1997. The cystic fibrosis transmembrane conductance regulator and ATP. Curr. Opin. Cell Biol. 9:547-552.

18. Naren, A.P., et al. 1997. Regulation of CFTR chloride channels by syntaxin and Munc18 isoforms. Nature. 390:302-305.

19. Naren, A.P., Quick, M.W., Collawn, J.F., Nelson, D.J., and Kirk, K.L. 1998. Syntaxin 1A inhibits CFTR chloride channels by means of domain-specific protein-protein interactions. Proc. Natl. Acad. Sci. USA. 95:10972-10977.

20. Short, D.B., et al. 1998. An apical PDZ protein anchors the cystic fibrosis transmembrane conductance regulator to the cytoskeleton. J. Biol. Chem. 273:19797-19801.

21. Kemp, B.E., et al. 1999. Dealing with energy demand: the AMP-activated protein kinase. Trends Biochem. Sci. 24:22-25.

22. Hardie, D.G., and Carling, D. 1997. The AMP-activated protein kinase. Fuel gauge of the mammalian cell? Eur. J. Biochem. 246:259-273.

23. Leclerc, I., Kahn, A., and Doiron, B. 1998. The 5'-AMP-activated protein kinase inhibits the transcriptional stimulation by glucose in liver cells, acting through the glucose response complex. FEBS Lett. 431:180-184.

24. Kurth-Kraczek, E.J., Hirshman, M.F., Goodyear, L.J., and Winder, W.W. 1999. 5' AMP-activated protein kinase activation causes GLUT4 translocation in skeletal muscle. Diabetes. 48:1667-1671.

25. Chen, Z.P., et al. 1999. AMP-activated protein kinase phosphorylation of endothelial NO synthase. FEBS Lett. 443:285-289.

26. Altschul, S.F., et al. 1997. Gapped BLAST and PSI-BLAST: a new generation of protein database search programs. Nucleic Acids Res. 25:3389-3402.

27. Estojak, J., Brent, R., and Golemis, E.A. 1995. Correlation of twohybrid affinity data with in vitro measurements. Mol. Cell. Biol. 15:5820-5829.

28. Gietz, R.D., and Woods, R.A. 1994. High efficiency transformation in yeast. In Molecular genetics of yeast: a practical approach. J.A. Johnston, editor. Oxford University Press. Oxford, United Kingdom. 121-134.

29. Pasyk, E.A., and Foskett, J.K. 1995. Mutant (DeltaF508) cystic fibrosis transmembrane conductance regulator $\mathrm{Cl}^{-}$channel is functional when retained in endoplasmic reticulum of mammalian cells. J. Biol. Chem. 270:12347-12350.

30. Michell, B.J., et al. 1996. Isoform-specific purification and substrate specificity of the $5^{\prime}$-AMP-activated protein kinase. J. Biol. Chem. 271:28445-28450.

31. Stapleton, D., et al. 1996. Mammalian AMP-activated protein kinase subfamily. J. Biol. Chem. 271:611-614.

32. Dyck,J.R.B., et al. 1996. Regulation of 5'-AMP-activated protein kinase activity by the noncatalytic $\beta$ and $\gamma$ subunits. J. Biol. Chem. 271:17798-17803.

33. Xiong, X.M., Bragin, A., Widdicombe, J.H., Cohn, J., and Skach, W.R. 1997. Structural cues involved in endoplasmic reticulum degradation 
of G85E and G91R mutant cystic fibrosis transmembrane conductance regulator. J. Clin. Invest. 100:1079-1088.

34. Crute, B.E., Seefeld, K., Gamble, J., Kemp, B.E., and Witters, L.A. 1998 Functional domains of the alpha1 catalytic subunit of the AMP-activated protein kinase. J. Biol. Chem. 273:35347-35354.

35. Jiang, Q.S., et al. 1998. Cystic fibrosis transmembrane conductance regulator-associated ATP release is controlled by a chloride sensor. J. Cell Biol. 143:645-657.

36. Hasegawa, H., et al. 1992. A multifunctional aqueous channel formed by CFTR. Science. 258:1477-1479.

37. Hardie, D.G., Carling, D., and Carlson, M. 1998. The AMP-activated/SNF1 protein kinase subfamily: metabolic sensors of the eukaryotic cell? Annu. Rev. Biochem. 67:821-855.

38. Jiang, R., and Carlson, M. 1997. The Snf1 protein kinase and its activating subunit, Snf4, interact with distinct domains of the Sip1/Sip2/Gal83 component in the kinase complex. Mol. Cell. Biol. 17:2099-2106

39. Prince, L.S., et al. 1999. Efficient endocytosis of the cystic fibrosis transmembrane conductance regulator requires a tyrosine-based signal. J. Biol. Chem. 274:3602-3609.

40. Marsh, M., and McMahon, H.T. 1999. The structural era of endocytosis. Science. 285:215-220.

41. Stapleton, D., et al. 1994. Mammalian 5'-AMP-activated protein kinase non-catalytic subunits are homologs of proteins that interact with yeast Snf1 protein kinase. J. Biol. Chem. 269:29343-29346.

42. Cheng, S.H., et al. 1990. Defective intracellular transport and processing of CFTR is the molecular basis of most cystic fibrosis. Cell. 63:827-834.

43. Carling, D., Clarke, P.R., Zammit, V.A., and Hardie, D.G. 1989. Purificaton and characterization of the AMP-activated protein kinase. Copurificaton of acetyl-CoA carboxylase kinase and 3-hydroxy-3 methylglutaryl-CoA reductase kinase activities. Eur. J. Biochem 186:129-136.
44. Weixel, K.M., and Bradbury, N.A. 2000. The carboxyl terminus of the cystic fibrosis transmembrane conductance regulator binds to AP-2 clathrin adaptors. J. Biol. Chem. 275:3655-3660.

45. Sharma, N., et al. 1999. The C terminus of SUR1 is required for trafficking of $\mathrm{K}_{\mathrm{ATP}}$ channels. J. Biol. Chem. 274:20628-20632.

46. Corton, J.M., Gillespie, J.G., Hawley, S.A., and Hardie, D.G. 1995. 5Aminoimidazole-4-carboxamide ribonucleoside. A specific method for activating AMP-activated protein kinase in intact cells? Eur. J. Biochem. 229:558-565.

47. Hall, R.A., et al. 1999. G protein-coupled receptor kinase $6 \mathrm{~A}$ phosphorylates the $\mathrm{Na}^{+} / \mathrm{H}^{+}$exchanger regulatory factor via a $\mathrm{PDZ}$ domain-mediated interaction. J. Biol. Chem. 274:24328-24334.

48. Fraser, I.D.C., and Scott, J.D. 1999. Modulation of ion channels: a "current" view of AKAPs. Neuron. 23:423-426.

49. Anderson, M.P., Sheppard, D.N., Berger, H.A., and Welsh, M.J. 1992. Chloride channels in the apical membrane of normal and cystic fibrosis airway and intestinal epithelia. Am. J. Physiol. 263:L1-L14.

50. Wilkinson, D.J., et al. 1997. CFTR activation additive effects of stimulatory and inhibitory phosphorylation sites in the R domain. Am. J. Physiol. 273:L127-L133.

51. Bradbury, N.A., and Bridges, R.J. 1994. Role of membrane trafficking in plasma membrane solute transport. Am. J. Physiol. 267:C1-C24.

52. Takahashi, A., Watkins, S.C., Howard, M., and Frizzell, R.A. 1996. CFTR-dependent membrane insertion is linked to stimulation of the CFTR chloride conductance. Am. J. Physiol. 271:C1887-C1894.

53. Haardt, M., Benharouga, M., Lechardeur, D., Kartner, N., and Lukacs, G.L. 1999. C-terminal truncations destabilize the cystic fibrosis transmembrane conductance regulator without impairing its biogenesis: a novel class of mutation. J. Biol. Chem. 274:21873-21877.

54. Brezillon, S., et al. 1997. ATP depletion induces a loss of respiratory epithelium functional integrity and down-regulates CFTR (cystic fibrosis transmembrane conductance regulator) expression. J. Biol. Chem. 272:27830-27838. 